\title{
Distally-based free anterolateral thigh flap with a modified vena comitans
}

\author{
Chan-Su Kang, Tae-Gon Kim \\ Department of Plastic and Reconstructive Surgery, Yeungnam University College of Medicine, Daegu, Korea
}

\begin{abstract}
With the recent development in microsurgery, the use of a perforator flap has been widely implemented. If the length of the ALT flap pedicle is insufficient despite adequate preoperative planning, pedicle length extension is necessary. We planned for a reverse ALT free flap using the distal vessel of the descending branch for pedicle length extension in the case of ALT perforator branch originating from the proximal portion of the descending branch. For the management of venous congestion, the distal venae comitantes were anastomosed to the proximal venous stump in an antegrade manner, successfully resolving the venous congestion. Modified reverse-flow ALT free flap, wherein the venae comitantes are anastomosed to the proximal vein stump, is a good option that allows for relatively simple pedicle extension within the same operative field when securing an adequate pedicle length is difficult because of the origin of the perforator from the proximal descending branch, unlike the initial surgical plan.
\end{abstract}

Keywords Lower extremity / Surgical flaps / Veins

Received: 29 Aug 2018 Revised: 23 Oct 2018 - Accepted: 26 Oct 2018

pISSN: 2234-6163 • elSSN: 2234-6171 • https://doi.org/10.5999/aps.2018.01018 • Arch Plast Surg 2019;46:84-87

\author{
Correspondence: Tae-Gon Kim \\ Department of Plastic and \\ Reconstructive Surgery, Yeungnam \\ University College of Medicine, 170 \\ Hyeonchung-ro, Nam-gu, Daegu \\ 42415, Korea \\ Tel: $+82-53-620-3483$ \\ Fax: +82-53-626-0705 \\ E-mail: kimtg0919@daum.net
}

This study was funded by a 2017 Yeungnam University research grant.

\section{INTRODUCTION}

With recent developments in microsurgery, perforator flaps have become widely used. After the first report on the anterolateral thigh (ALT) flap concept by Song et al. [1] in 1984, Luo et al. [2] described the anatomy and application of the ALT flap in 1985. Since then, the ALT flap has become the most commonly used perforator flap in plastic surgery owing to its versatility, ease of use, and minimal donor site morbidity [3-6]. If the length of the ALT flap pedicle is insufficient despite adequate preoperative planning, pedicle length extension is required. Recent studies have reported pedicle length modification within the ALT operative field [7-10]. We attempted pedicle length extension with the distal descending branch in a case of insufficient pedi- cle length in the proximal direction because the perforator emerged from a quite proximal portion of the descending branch. When temporary clamping of the proximal vessel was attempted, venous congestion occurred. As a corrective measure, the vena comitans was transferred to the proximal stump and anastomosis was performed to allow venous blood to flow in the forward direction, and the flap circulation stabilized.

\section{CASE}

A 60 -year-old man presented with a $16 \times 6 \mathrm{~cm}^{2}$ open wound with an accompanying tibiofibular fracture due to a traffic accident. Free flap coverage was planned, with a necessary pedicle length of $14 \mathrm{~cm}$ from the middle of the flap to the recipient ves- 
sel and $9 \mathrm{~cm}$ from the flap margin. A $20 \times 6 \mathrm{~cm}^{2}$ ALT flap from the patient's ipsilateral thigh was planned. The perforator that branched at the proximal descending branch of the lateral circumflex femoral artery was found intraoperatively (Fig. 1). The length of the branched perforator was $7 \mathrm{~cm}$, and the available length of the descending branch on the proximal side was $2 \mathrm{~cm}$, so the estimated pedicle length was $9 \mathrm{~cm}$ (Fig. 2). For pedicle length extension, dissection was performed on the distal aspect, which resulted in obtaining $7 \mathrm{~cm}$ of the distal descending branch.

\section{Fig. 1. Perforator branched from proximal portion}

Pedicle of the anterolateral thigh flap: distal (a) and proximal (b) parts of the distal vena comitans. A pedicle branching from the proximal portion of the descending branch of the lateral circumflex femoral artery to the flap was confirmed. The distal branch was obtained for pedicle extension.

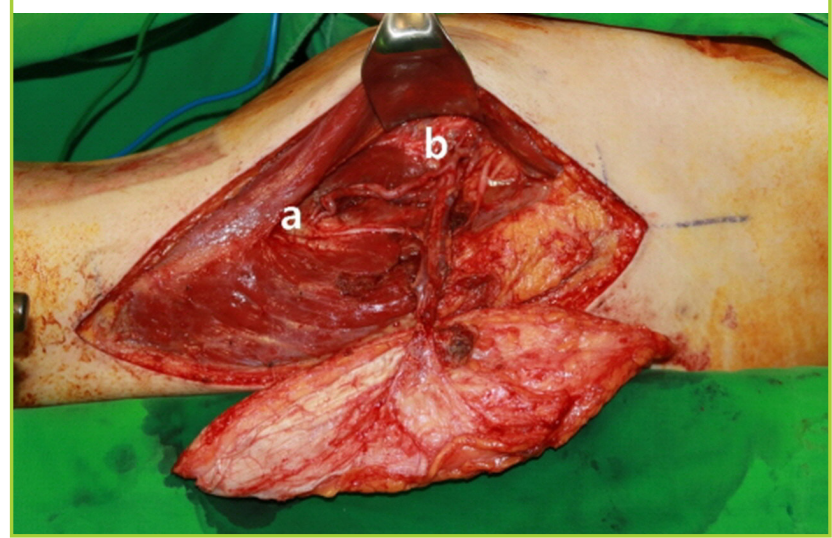

Thus, we were able to obtain a reverse ALT free flap with a total pedicle length of $14 \mathrm{~cm}$ in the distal direction. Before elevating the reverse ALT free flap, we clamped the proximal descending branch to check for flap circulation. We confirmed that flap congestion was present due to impaired retrograde flow through the valves of the vena comitans in the distal direction. To resolve flap congestion, one of the venae comitantes of the distal descending branch was isolated and connected to the proximal venous stump in an antegrade manner (Fig. 3). When connecting a 1.5-mm vena comitans of the distal descending branch with the 2-mm proximal venous stump, the diameter discrepancy of

\section{Fig. 2. Elevated anterolateral thigh flap}

Harvested free anterolateral thigh (ALT) flap: distal (a) and proximal (b) parts of the distal vena comitans. A free ALT flap was harvested from the patient's ipsilateral thigh.

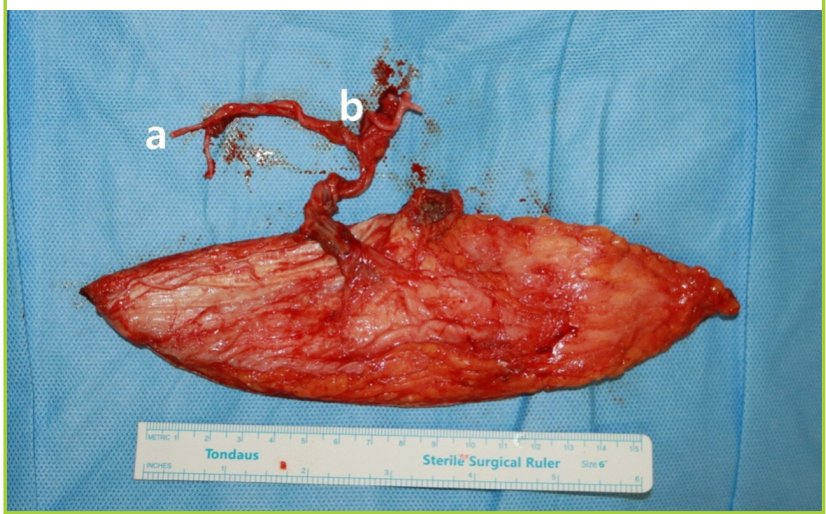

Fig. 3. Transposition of venae comitantes

Scheme of the distally-based anterolateral thigh free flap with a modified vena comitans: distal (a) and proximal (b) parts of the distal vena comitans. Venous valves are indicated by a yellow line. (A) The length of the perforator was $7 \mathrm{~cm}$, that of the proximal descending branch was $2 \mathrm{~cm}$, and that of the distal descending branch was $7 \mathrm{~cm}$, so the antegrade pedicle length would be $9 \mathrm{~cm}$. Venous congestion occurred when proximal descending branch was clamped because of the reversed direction of the venous valves. (B) An approximately 6 -cm long segment of the distal vena comitans was transferred to the proximal stump to allow venous flow in the forward direction. (C) The final pedicle length was $15 \mathrm{~cm}$. The venous blood was able to flow without reversing the valves.

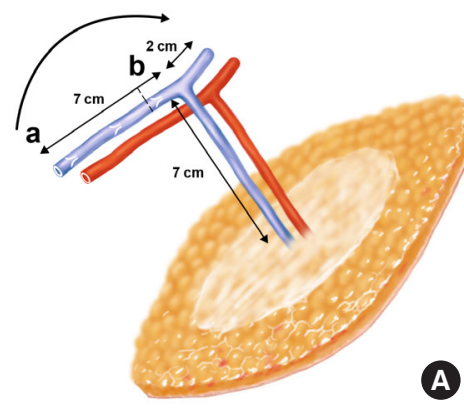

A
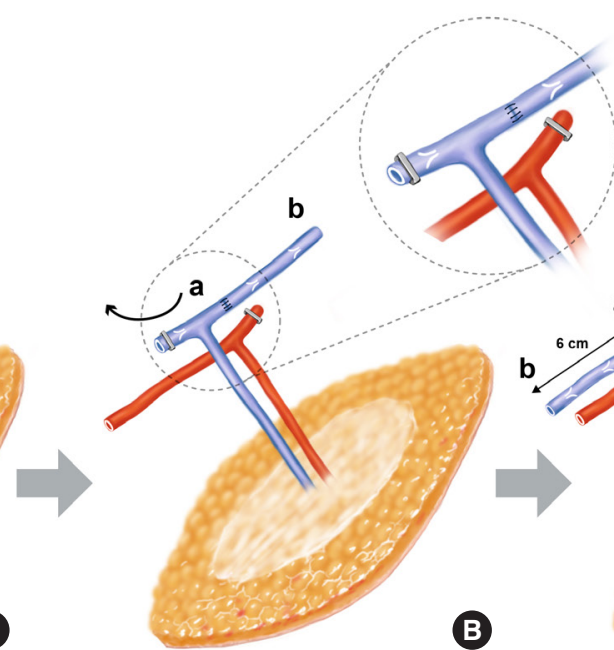

....

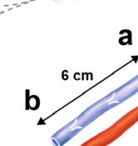

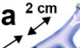

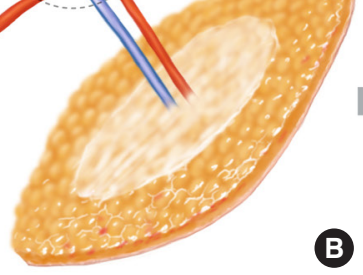

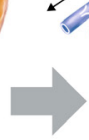

B

( 


\section{Fig. 4. Antegrade venous flow after anastomosis}

After anastomosis: distal (a) and proximal (b) parts of the distal vena comitans. The distal vena comitans was connected to the proximal venous stump and anastomosed (red oval, arterial microanastomosis site and; blue ovals, venous micro-anastomosis sites).

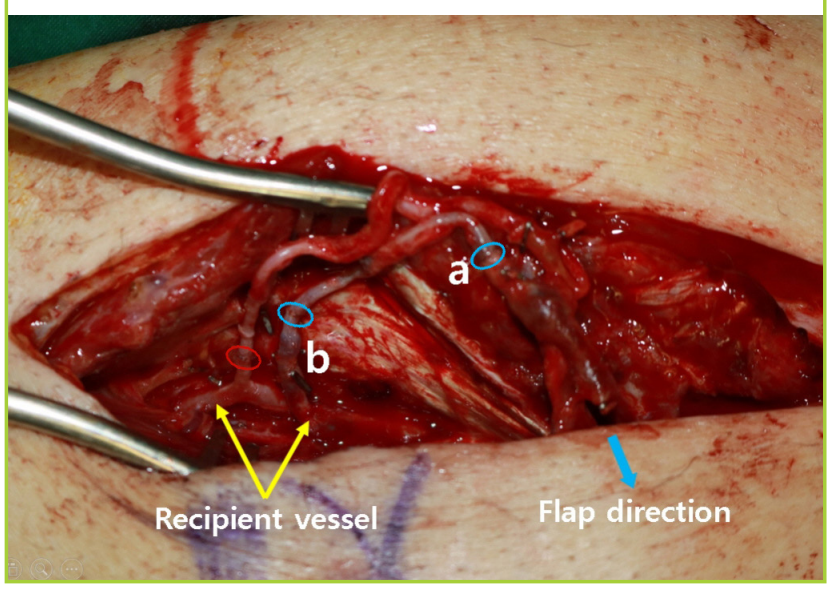

the venous anastomosis was not significant (Fig. 4). A modified ALT flap pedicle $15 \mathrm{~cm}$ in length was formed to prevent venous congestion and to ensure adequate length to reach the recipient vessel. As a result, the direction of the arterial flow to the flap was retrograde, and the venous flow was antegrade. To evaluate the flap condition after surgery, the flap color was checked and the pin-prick test and Doppler ultrasonography were performed. For 2 days after surgery, the flap status was maintained, with no findings of congestion or insufficiency. Examinations during the 3-month follow-up revealed that the flap was maintained without complications.

\section{DISCUSSION}

When a pedicle is too short, excessive dissection in the proximal direction of the descending branch to obtain a long pedicle for the ALT flap is not recommended because of the possibility of size discrepancy at anastomosis. Previously described methods for pedicle lengthening include vein grafts, arteriovenous loops, and arteriovenous bundle grafts. Recent studies have reported ALT flap pedicle length extension within the operative field [7$10]$.

Many authors have reported the successful use of a reversed island ALT flap [11-14], but there is a possibility of venous congestion [15]. There have been reports of persistent congestion requiring additional venous drainage in reversed island ALT flaps. Similarly, we report this case of an ALT free flap using the distal vessel of the descending branch for pedicle length extension when the perforator emerged at the proximal portion of the descending branch. For the management of venous congestion, the distal vena comitans was antegradely anastomosed to the proximal venous stump.

The distally-based ALT free flap with modification of the vena comitans is a good option that enables relatively simple pedicle extension within the same operative field when securing an adequate pedicle length is difficult because of the proximal location of the perforator in the descending branch.

\section{NOTES}

\section{Conflict of interest}

No potential conflict of interest relevant to this article was reported.

\section{Ethical approval}

The study was approved by the Institutional Review Board of Yeungnam University Medical Center (IRB No. 2018-08-049). Written informed consent was obtained.

\section{Patient consent}

The patient provided written informed consent for the publication and the use of his images.

\section{ORCID}

Chan-Su Kang https://orcid.org/0000-0002-2005-6779

Tae-Gon Kim https://orcid.org/0000-0002-6738-4630

\section{REFERENCES}

1. Song YG, Chen GZ, Song YL. The free thigh flap: a new free flap concept based on the septocutaneous artery. Br J Plast Surg 1984;37:149-59.

2. Luo LS, Gao JH, Chen LF. A new free skin flap-anterolateral femoral flap: it's anatomy and clinical application. Chin J Plast Surg Burns 1985;1:50.

3. Koshima I, Fukuda H, Yamamoto H, et al. Free anterolateral thigh flaps for reconstruction of head and neck defects. Plast Reconstr Surg 1993;92:421-8.

4. Wei FC, Celik N, Chen HC, et al. Combined anterolateral thigh flap and vascularized fibula osteoseptocutaneous flap in reconstruction of extensive composite mandibular defects. Plast Reconstr Surg 2002;109:45-52.

5. Koshima I, Nanba Y, Tsutsui T, et al. Perforator flaps in lower extremity reconstruction. Handchir Mikrochir Plast Chir 2002;34:251-6.

6. Koshima I, Nanba Y, Tsutsui T, et al. New anterolateral thigh perforator flap with a short pedicle for reconstruction of defects in the upper extremities. Ann Plast Surg 2003;51:30-6. 
7. Han HH, Jun D, Mun GH, et al. Modification of pedicle lengthening through perforator transposition in free anterolateral thigh flaps with pedicle size discrepancy: a case report. Microsurgery 2018;38:94-7.

8. Han HH, Choi EJ, Oh DY, et al. The usefulness of microsurgical pedicle lengthening in free anterolateral thigh flaps. Microsurgery 2016;36:559-66.

9. Gao SH, Feng SM, Chen C, et al. A new recipient artery for reconstruction of soft-tissue defects in the lower limb with a free anterolateral thigh flap: the reversed descending branch of the lateral femoral circumflex artery. Plast Reconstr Surg 2012;130:1059-65.

10. Huang YH, Hsieh TY, Lai CS, et al. In situ pedicle lengthening of the anterolateral thigh flap. Plast Reconstr Surg 2014; 133:85e-87e.

11. Pan SC, Yu JC, Shieh SJ, et al. Distally based anterolateral thigh flap: an anatomic and clinical study. Plast Reconstr Surg 2004; 114:1768-75.

12. Zhou G, Zhang QX, Chen GY. The earlier clinic experience of the reverse-flow anterolateral thigh island flap. Br J Plast Surg 2005;58:160-4.

13. Gravvanis AI, Tsoutsos DA, Karakitsos D, et al. Application of the pedicled anterolateral thigh flap to defects from the pelvis to the knee. Microsurgery 2006;26:432-8.

14. Komorowska-Timek E, Gurtner G, Lee GK. Supercharged reverse pedicle anterolateral thigh flap in reconstruction of a massive defect: a case report. Microsurgery 2010;30:397400.

15. Kim IK, Kim TG, Lee JH, et al. The clinical course of reverse-flow anterolateral thigh flap: need to prepare for venous congestion and salvage operation. Arch Plast Surg 2012;39:262-4. 